\title{
El conocimiento especializado de um maestro de primaria cuando enseña la resta
}

\author{
The specialized knowledge of a primary teacher when working on subtraction \\ Mercedes Alberca Bonilla ${ }^{1}$ \\ Luis Carlos Contreras González ${ }^{2}$
}

\begin{abstract}
Resumo
Esta pesquisa visa compreender o conhecimento especializado que um professor de matemática possui em relação à subtração, em cujo processo de ensino utiliza algoritmos abertos baseados em números (método $\mathrm{ABN}$ ), utilizando o modelo Mathematics Teacher Specialized Knowledge (MTSK) para sua análise. Para o efeito, foi realizado um estudo de caso de um professor primário do segundo ano de uma Escola Concertada de Huelva. A partir das transcrições das gravações de quatro atividades em que a subtração é trabalhada, bem como de uma posterior entrevista semiestruturada, foram obtidas evidências e indícios que mostram que o professor possui conhecimento especializado em subdomínios de conhecimento de conteúdo e conhecimento didático conteúdo, o que nos permite presumir uma relação entre esse conhecimento e a formação docente no método ABN.
\end{abstract}

Palavras-chave: Resta; Conocimiento Especializado; Algoritmos Abiertos Basados en Números; Estudio de Caso.

\begin{abstract}
This research focuses on understanding the specialized knowledge that a mathematics teacher has in relation to subtraction, in whose teaching process uses Open Algorithms Based on Numbers (ABN method), using the Mathematics Teacher Specialized Knowledge (MTSK) model to your analysis. Therefore, it has carried out a case study about a second primary teacher from Concerted School in Huelva. On the other hand, the transcripts of the recordings of four activities in which the subtraction is worked, as well as from a subsequent semistructured interview have obtained evidences and indications that show the teacher has specialized knowledge in subdomains about knowledge of contents and didactic knowledge, which allow us to presume a relationship between theses knowledges and teacher training in the ABN method.
\end{abstract}

Keywords: Subtraction; Specialized Knowledge; Open Algorithm Based on Numbers; Case Study.

\section{Submetido em: 14/09/2020 - Aceito em: 01/02/2021 - Publicado em: 27/05/2021}

1 Doutoranda em Investigaçao da Didáctica da Matemática pela Universidade de Huelva, Espanha. Email: mercheeab97@gmail.com. ORCID: https://orcid.org/0000-0002-3691-0589

2 Doutor em Psicopedagogía pela Universidade de Huelva, Espanha. Catedrático de Didáctica de la Matemática da Universidade de Huelva, Espanha. Email: 1carlos@ uhu.es. ORCID: https://orcid.org/0000-0002-0044-2365 


\section{Introducción}

Ante las dificultades detectadas en los alumnos/as en relación con el cálculo aritmético a lo largo del tiempo, parece necesario un cambio en su enseñanza, más allá del uso de métodos repetitivos y poco significativos; cambios que supongan la implantación de nuevas estrategias y técnicas (Fuson, 1992; Carpenter, Franke, Jacobs \& Fennema, 1996; Brown \& Burton, 1978). Quizás por ello han surgido nuevas formas de trabajar el cálculo en el aula, como es el Algoritmo Abierto Basado en Números (ABN), que ofrecen una diversidad en la forma de proceder en el cálculo y multitud de técnicas que se basan en las necesidades del alumnado (Bracho-López, 2013).

Aunque $\mathrm{ABN}$ es reciente, los fundamentos de las técnicas que este método ofrece ya fueron expuestos años atrás, por autores (Castro, Rico \& Castro, 1987; Maza, 1991) que resaltaron la importancia del Sistema Numérico Decimal y del uso de estrategias que facilitasen el cálculo. Sea cual sea el nombre de estas técnicas, parece evidente que su conocimiento, por parte del profesor, se hace fundamental para que la enseñanza de la resta se lleve a cabo adecuadamente.

En esta investigación se explora el conocimiento de un docente que ya mostró su interés por colaborar con la Universidad de Huelva, en relación con la realización de investigaciones que pusiesen en relevancia las potencialidades y problemáticas que ofrece el método $\mathrm{ABN}$ en el ámbito de la enseñanza de las matemáticas. Además, se le puede considerar como docente con experiencia dados los años que lleva utilizando $A B N$ en sus clases y su asistencia frecuente a encuentros en los que se debate el uso del método.

En la exploración del conocimiento del profesor se han venido utilizando diferentes modelos analíticos, la mayoría de ellos inspirados en los trabajos seminales de Shulman (1986, 1987). Como se expondrá más adelante, nuestro trabajo utilizará el modelo analítico del conocimiento especializado del profesor de matemáticas (MTSK, de sus siglas en inglés)(Carrillo et al. 2018).

Por consiguiente, las preguntas de investigación que se plantean son las siguientes:

- ¿Qué conocimiento especializado muestra un maestro de Primaria cuando trabaja la resta en segundo curso?

- ¿Cuáles de esos conocimientos pueden estar relacionados con su formación en ABN?

En relación con estas preguntas, los objetivos de esta investigación son los siguientes: (a) identificar los distintos elementos del conocimiento especializado que el maestro pone en juego cuando trabaja la resta con sus estudiantes y (b) identificar cuáles de esos conocimientos tienen relación con su formación con $\mathrm{ABN}$. 


\section{Fundamentos teóricos}

Esta investigación se enmarca dentro del conocimiento del professor de matemáticas y, para ello, toma como soportes teóricos el modelo analítico del conocimiento especializado del profesor de matemáticas (en adelante, MTSK), que a su vez se constituye como herramienta de análisis, y el método ABN (Algoritmos Abiertos Basados en Números). En cuanto a este segundo, consideramos sus principios y la justificación de su aparición (sus antecedentes). De los contenidos que reformula el método ABN se incluyen las técnicas utilizadas para la resta, pues este será el contenido que se tomará como base para saber el conocimiento especializado que posee el docente. El otro pilar de este trabajo se centra en el conocimiento del profesor. Para el análisis de tal contenido y como fundamentación teórica se ha incluido el MTSK que nos proporciona un instrumento de análisis para el conocimiento especializado.

\section{Antecedentes de ABN}

Ha habido una gran evolución en las necesidades que presenta el alumnado de los centros escolares. Ante las mismas, surge la necesidad de una transformación metodológica que dé respuesta a los intereses de los discentes. Concretamente, y en lo que a las matemáticas se refiere, hace ya casi medio siglo que Ablewhite y Paret (1971) cuestionaron la eficacia de los métodos de enseñanza del cálculo aritmético escolar, tras observar los numerosos problemas que el alumnado presentaba con el uso mecánico de sus algoritmos.

Según Bracho-López, Gallego-Espejo, Adamuz-Povedano y Jiménez-Fanjul (2014), las evoluciones en los métodos de enseñanza de las matemáticas son necesarias. Muchos de los cambios paulatinos que se han dado evidenciaban la necesidad de cambios más profundos que saldaran los problemas que caracterizan esta forma de enseñar, debido a la irracionalidad que supone el aprendizaje memorístico y sin comprensión de sus algoritmos.

De estas dificultades que presentan los métodos tradicionales de cálculo (basados en algoritmos comprimidos) y de las necesidades que aún quedaban por resolver en el alumnado, surge, entre otros métodos, el Algoritmo Abierto Basado en Números, un método que trata de aproximarse a las necesidades actuales de los centros educativos (Martínez, 2011).

\section{Algorítmos Abiertos Basados en Números (ABN)}

En el año 2000 nace una alternativa a los algoritmos convencionales, creada por Jaime Martínez, los Algoritmos Abiertos Basados en Números (ABN, en adelante). Desde su creación, este autor realiza modificaciones que van perfeccionando el método, siendo en el año 2010 cuando publica su método definitivo, haciendo explícita la forma de incluirlo en clase (Martínez, 2011).

Según Martínez (2011), el objetivo fundamental del ABN es poner el foco de atención en los números (en la estructura del Sistema de Numeración Decimal; en adelante, SND) y no en los algoritmos, realizando cálculos más abiertos (y comprensibles) siendo el estudiante 
quien elige los pasos que necesita dar para resolverlo ${ }^{3}$. Gracias al desarrollo del método, se facilita, en consecuencia, la mejora en varios ámbitos: cálculo mental, resolución de problemas y actitud del alumnado ante el aprendizaje matemático.

No se pone el énfasis en las operaciones con simples cifras, sino que se dota de un contexto trabajando, en su lugar, con problemas contextualizados, cobrando importancia, de esta forma, como se ha señalado, el SND (Bracho-López, Adamuz-Povedano, GallegoEspejo \& Jiménez-Fanjul, 2014). Además, según Bracho-López et al., (2014), trabajar con números (con el SND) lleva consigo dotar a las cifras de un tratamiento distintivo en función de su valor relativo (según se trate de unidades, decenas o centenas), y no solo de su valor absoluto, como de forma mecánica sucede con los algoritmos convencionales. Así, y en relación con la contextualización, ABN propone trabajar con materiales manipulables (palillos, monedas de cartón...) (Canto, 2014).

Hay una gran diferencia del método tradicional o Cálculo Basado en Cifras (CBC, en adelante) en cuanto a las operaciones que se realizan. La resta en $\mathrm{ABN}$, según Martínez (2018), se puede llevar a cabo mediante cuatro formas diferentes, la elección dependerá de lo que el alumnado necesite realizar (ver Tabla 1). Además, en ella no existen las llevadas, ni los ceros. Así, no implica "quedarse en la cabeza" con ningún dato, estando todos los pasos presentes en el cálculo realizado (Martínez, 2018).

Tabla 1 - Síntesis del proceso de resta en ABN

\begin{tabular}{cc}
\hline Detracción & RESTA \\
$\begin{array}{c}\text { Comsiste en ir quitando la misma cuantía de las dos cantidades con las que se } \\
\text { trabaja hasta que una de ellas llega a cero. } \\
\text { Escalera } \\
\text { ascendente }\end{array}$ & $\begin{array}{r}\text { Permite señalar cuál de las cantidades dadas es mayor y cuál es menor. } \\
\text { cantidad que se quiere llegar (cantidad deseada), y es en la primera columna } \\
\text { donde tienen que ir sumando hasta que lleguen a esa cantidad. }\end{array}$ \\
$\begin{array}{c}\text { Escalera } \\
\text { descendente }\end{array}$ & Cambio de sentido de la operación anterior, es decir, se busca hacer una \\
&
\end{tabular}

Fuente: adaptado de Martínez, (2011).

La diferencia de $\mathrm{ABN}$ respecto al $\mathrm{CBC}$ es que, como se ha señalado anteriormente, es el estudiante quien decide el orden realizando los pasos que estime convenientes para su resolución (Martínez, 2011).

ABN podría considerarse un procedimiento para la realización de cálculos con significado, frente al cálculo mecánico sin significado que suele caracterizar al CBC. Su conocimiento por parte del profesor implica su comprensión, así como el conocimiento de las

${ }^{3}$ Al más puro estilo del cálculo mental 
propiedades que fundamentan el cálculo y cabe pensar que conllevaría a un uso comprensivo por parte de su alumnado. Por otro lado, también podría considerarse un recurso. En ambos casos estamos ante dos elementos del conocimiento del profesor desde la perspectiva que expondremos a continuación.

\section{Mathematics teacher specialized knowledge (MTSK)}

En las últimas décadas ha cobrado interés comprender qué conocimiento posee un profesor acerca de un contenido. En la literatura de la investigación no fue hasta Shulman (1986) cuando se hizo explícita la falta de investigación existente en este ámbito. Shulman acuña el concepto de Conocimiento Didáctico del Contenido como un paradigma olvidado hasta ese momento. El denominado Pedagogical Content Knowledge (PCK, traducido como conocimiento didáctico del contenido) es de gran importancia pues constituiría el conjunto de saberes que un profesor posee para su enseñanza, es una amalgama de la didáctica y el contenido a enseñar que hace que un profesor sea capaz de adaptar un contenido para hacerlo comprensible y según los interés y capacidades de los alumnos/as (Shulman, 1986; Shulman, 1987). Por ello, Shulman (1986) transforma la conocida frase de Bernard Shaw, en la que parece desprestigiarse la labor docente ("quien sabe hace, quien no, enseña", p.4) en "quien sabe hace, quien comprende, enseña" (p.5), enfatizando la comprensión del contenido como clave para su transformación.

Aunque Shulman (1986) ya mencionó la importancia del conocimiento didáctico del contenido, no creó explícitamente ningún modelo de análisis para el mismo, siendo Ball, Thames y Phelps (2008) los pioneros en proporcionar un modelo analítico. En este estudio se empleará el Mathematics Teachers' Specialised Knowledge (MTSK) al ser un modelo de análisis que permite estudiar el conocimiento especializado del profesor que posee para la enseñanza de las matemáticas (Carrillo, Contreras \& Montes, 2013). Este modelo ha surgido en la Universidad de Huelva y busca mejorar y dar respuesta a las dificultades que ofrece el anterior modelo desarrollado por Ball, Thames y Phelps (2008), el MKT (Mathematical Knowledge for Teaching), sobre todo en cuanto a la diferenciación entre conocimiento común y especializado (MTSK consiera todo el conocimiento del profesor especializado), que desde la perspectiva analítica parece disgregar, por ejemplo, el conocimiento de las dificultades y errores de los estudiantes, de las razones o fundamentos matemáticos que nos permiten comprender la naturaleza de dichos errores, ofreciendo un modelo que es especializado en su conjunto (Carrillo, Climent, Contreras \& Muñoz Catalán, 2013). 


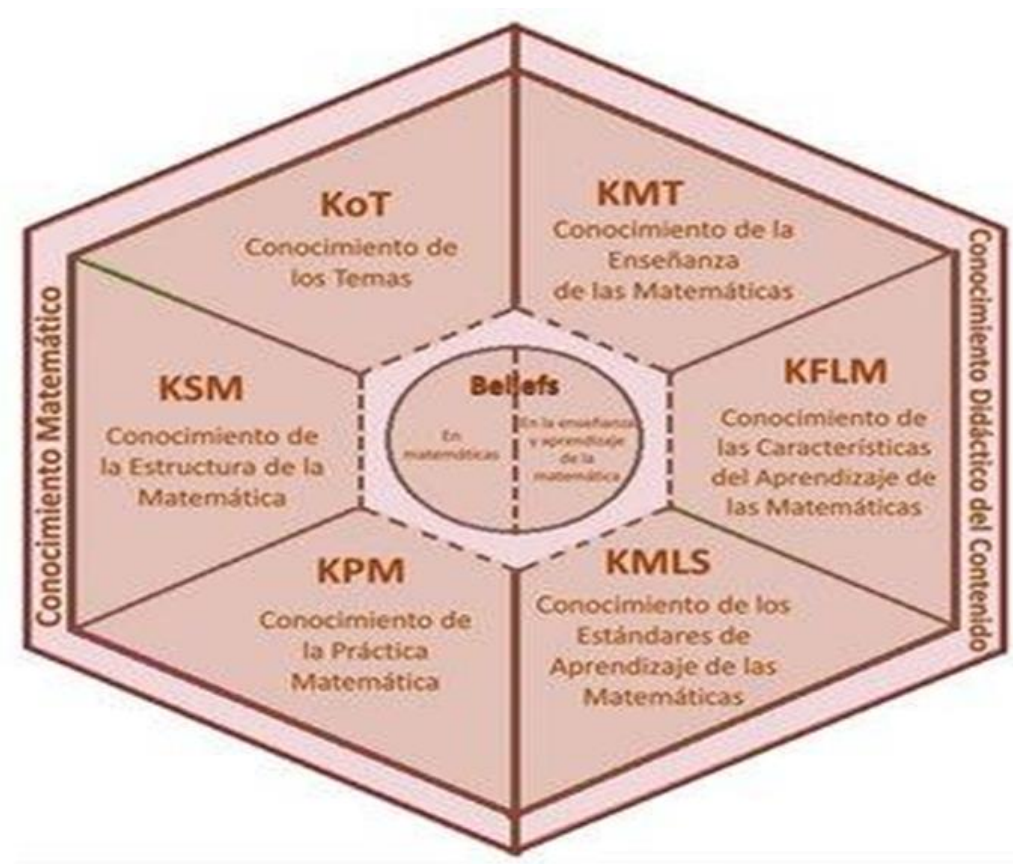

Figura 1 - Subdominios del MTSK

Fuente: (Carrillo et al, 2013).

Dentro de este modelo, además del dominio de las creencias, como un elemento que influye en el conocimiento del profesor y, por tanto, en cada uno de los subdominios presentes en el modelo (Carrillo et al., 2013), hay dos dominios de conocimiento: el Conocimiento Matemático (MK) y el Conocimiento Didáctico del Contenido (PCK). Según Carrillo, et al. (2013) se trata de dos aspectos que Shulman (1986) ya abordó y que quedan recogidos en este modelo con indicadores precisos para su análisis.

El Conocimiento Matemático (MK) se divide, a su vez, en tres subdominios con sus correspondientes categorías asociadas a cada uno que facilitarían su análisis (Carrillo et al., 2013; Aguilar, 2013):

- Conocimiento de los Temas (KoT): aborda el conocimiento del contenido matemático que un profesor posee. Incluye un conocimiento profundo sobre los significados, definiciones, propiedades o ejemplos del tema a tratar. En él encontramos cuatro categorías que servirían para analizarlos elementos que encontramos en el mismo: Definiciones, Propiedades y Sus Fundamentos; Procedimientos; Registros de Representación y Fenomenología y Aplicaciones.

- Conocimiento de la Estructura Matemática (KSM): se trata de los lazos de conexión que un profesor/a establece entre dos contenidos. Estas relaciones pueden ser, según sus categorías: Conexiones de Complejización; Conexiones de Simplificación; Conexiones Transversales y Conexiones Auxiliares.

- Conocimiento de la Práctica Matemática (KPM): se trata del conocimiento de los procedimientos que llevarían a construir o validar un resultado matemático, así como 
el conocimiento de la forma de comunicar matemáticas. En este subdominio aún no existen categorías, pero si hay una serie de condiciones aceptadas que se encuadrarían dentro del mismo, como pueden ser las formas de validación y demostración.

En el Conocimiento Didáctico del Contenido (PCK) se encuentran los siguientes subdominios (Carrillo et al.,2013; Aguilar, 2013):

- Conocimiento de la Enseñanza de las Matemáticas (KMT): en él se engloba el conocimiento que debe tener un profesor sobre los diferentes recursos, ejemplos, materiales en relación con un contenido matemático y la adecuación de su uso en la enseñanza. En este subdominio encontramos tres categorías: Teorías Sobre Enseñanza; Recursos Materiales y Virtuales y Estrategias, Técnicas, Tareas y Ejemplos.

- Conocimiento de las Características de Aprendizaje de las Matemáticas (KFML): se trata del conocimiento del profesor sobre las características de aprendizaje de los alumnos/as en relación con un contenido. Encontramos cuatro categorías: Teorías sobre Aprendizaje; Fortalezas y Dificultades; Formas de Interacción con un Contenido Matemático e Intereses y Expectativas.

- Conocimiento de los Estándares de Aprendizaje de las Matemáticas (KMLS): se trata de conocer lo que indican los distintos elementos curriculares en relación con los logros de aprendizaje que un alumno/a debe alcanzar en un nivel determinado, también implica el conocimiento sobre lo que las investigaciones en educación matemática indican al respecto. Para analizar este conocimiento se subdivide en tres categorías: Expectativas de Aprendizaje; Nivel de Desarrollo Conceptual o Procedimental Esperado y Secuenciación con los Temas Anteriores y Posteriores.

Por tanto, el presente modelo (MTSK) es un instrumento de análisis que sirve para conocer y profundizar sobre el conocimiento que posee un docente en un contenido matemático específico. Este instrumento será utilizado para comprender el conocimiento que posee un maestro sobre la resta; a través de una entrevista realizada tras la observación, indagaremos sobre su formación em $\mathrm{ABN}$ para conjeturar acerca de las posibles relaciones entre ese conocimiento y su vinculación con el método ABN, en el sentido de su influencia en el conocimiento de tal contenido.

\section{Metodología}

Esta investigación se sitúa en el paradigma interpretativo pues, tal y como señala Muñoz-Catalán (2009), con este paradigma se busca interpretar la realidad en su contexto; en este caso, se pretende comprender el conocimiento especializado que despliega un docente en su aula al enseñar la sustracción. Además, se trata de un estudio con enfoque cualitativo pues en él se emplean grabaciones y entrevistas que permiten obtener una visión interpretativa y naturalista de la realidad (Denzin \& Lincoln, 2000, citado en Muñoz-Catalán, 2009).

En cuanto al método empleado ha sido el estudio de caso, que Bassey (1999) define 
como el estudio de una singularidad conducida en profundidad en entornos naturales (p.47). De los tres tipos de estudio de caso que señala Stake (2000) esta investigación se encuadraría en el estudio de caso instrumental pues es el que permite comprender más profundamente un tema, a partir del estudio de un caso particular, en este caso, el de Bartolomé.

Bartolomé es un maestro que, tras sus 22 años de experiencia como docente, lleva 8 años usando el método ABN en las aulas de un colegio concertado. Para mejorar su dominio del método, ha asistido a más de doce cursos para obtener formación en el mismo. Además, participa activamente para este con la creación de un blog donde aporta su experiencia como docente que trabaja con $\mathrm{ABN}$ y la elaboración de varios materiales didácticos.

Para la recogida de información se han utilizado las grabaciones de vídeo que se han llevado a cabo en los meses de enero y febrero (de 2020) en las sesiones de clase de matemáticas impartidas por Bartolomé en segundo de Primaria. De la transcripción literal de las mismas se han seleccionado las actividades en cuya resolución implicaba la realización de la resta. Además, posteriormente, se le ha realizado una entrevista o con el fin de profundizar más sobre su conocimiento especializado que pone en juego en estas actividades y, sobre todo, para obtener evidencias sobre si su conocimiento, puesto de manifiesto, tiene relación con su formación en $\mathrm{ABN}$.

En lo que al análisis de datos respecta, el instrumento de análisis empleado ha sido el MTSK, pues permite conocer e interpretar el conocimiento especializado que pone en juego un docente cuando enseña un contenido específico de matemáticas (la sustracción). Se trata de un instrumento que, como ya se ha indicado, presenta diferentes subdominios y categorías que facilitarían el análisis de la información y que se lleva utilizando desde el año 2013 en diferentes trabajos de investigación, con diferentes niveles educativos y contenidos curriculares. Una vez se trascribe la información (tanto la que proviene de la observación, como de la entrevista), se seleccionan aquellos episodios que parecen obtener datos relevantes y, usando el sistema de categorías de MTSK, se organizan esos datos en las categorías señaladas. Todo el proceso de análisis se realiza con triangulación de interpretaciones (investigadora y coinvestigador).

\section{Análisis de los resultados}

En este apartado se va a realizar el análisis del conocimiento especializado del maestro sobre la sustracción presente en cuatro actividades y de las que se han obtenido diferentes evidencias. En todo momento el docente ha trabajado con el libro de texto de una de las editoriales que trabaja $\mathrm{ABN}$, por lo que estas actividades forman parte de él. Para ello, se resaltarán los subdominios que el docente ha puesto en juego en su proceso de enseñanzaaprendizaje de tal contenido; así como su relación de este conocimiento con la formación en el método ABN. Antes de entrar en el análisis, presentaremos cada una de las atividades. 


\section{ACTIVIDAD 1}

El docente pide a los/as alumnos/as que saquen unos materiales que servirán de apoyo para la comprensión de esta actividad (círculos, medios círculos y cuartos de círculo de cartulina que representan la hora, media hora y cuarto de hora respectivamente). Con ayuda de estos materiales y la rejilla (una "tabla" que facilita al alumnado la realización de las operaciones viendo de forma explícita el proceso), realizarán la operación de la resta relacionada con un problema que el docente le ha planteado: "Estoy en mi casa a las 15:30 y voy a la compra, llego a mi casa a las 17:00. ¿Cuánto tiempo pasó?”

\section{ACTIVIDAD 2}

El maestro le presenta a los discentes un problema en el que aparecen dos productos, el precio de uno y la diferencia de precio respecto al otro, los alumnos/as deben calcular, con la ayuda de los datos dados, el coste de uno de los productos. Este es el enunciado "El móvil vale 684 euros. El móvil es 186 euros más caro que la tableta. ¿Cuánto vale la tableta?”

\section{ACTIVIDAD 3}

El docente les presenta a los/as alumnos/as un problema en el que un hombre tiene una cuantía de dinero y necesita llegar hasta otra cantidad para poder comprar un producto; la técnica más idónea que los/as niños/as deben usar para resolverlo adecuadamente es la escalera ascendente. Su enunciado es el siguiente "Tienes 4,38 euros. ¿cuánto te falta para llegar a 10 ?"

\section{ACTIVIDAD 4}

Bartolomé les pide a los discentes que realicen una actividad en la que se le da una cantidad de dinero (que viene representado con números decimales -2,82-), los alumnos/as deberán partir de esa cantidad para llegar a otra cuantía dada. Para explicar esta idea, dado que los alumnos/as encuentran dificultades por los decimales, el docente usa tanto colores en los diferentes números como monedas de cartón. El enunciado dice lo siguiente "Tengo 2,82 euros, ¿cuánto me falta para llegar a 10 euros?" (Aunque esta actividad tenga la misma forma de resolución que la anterior en ella el docente emplea estrategias y recursos diferentes en las que se han mostrado evidencias de conocimiento distintas al anterior como veremos a continuación).

En el análisis del desarrollo de estas actividades se han encontrado conocimiento en dos subdominios relacionados con el Conocimiento de las Matemáticas (MK) concretamente son Conocimiento de los Temas (KoT) y Conocimiento de la Estructura de las Matemáticas (KSM) y otros dos subdominios relacionados con el Conocimiento Didáctico del Contenido (PCK) estos son el Conocimiento de las Características de Aprendizaje de las Matemáticas (KFLM) y el Conocimiento de la Enseñanza de las Matemáticas (KMT). A continuación, se presenta el conocimiento especializado del docente dividido en los subdominios mencionados. 


\section{Dominio del Conocimiento Matemático (MK)}

\section{Análisis del Conocimiento de los Temas (KoT)}

Las unidades de información encontradas, que se muestran a continuación, se encuentran en la categoría Procedimientos. En lo que respecta a este subdominio, el docente (al que en las transcripciones denominaremos B) muestra conocimiento de las características de una resta; sabe que, si a una cantidad le restas otra, el resultado es la diferencia de ambas, tiene conocimiento sobre su significado, este conocimiento estaría relacionado con el ¿Cómo se hace? Un descriptor de la categoría de los Procedimientos. El profesor conoce una de las formas de trabajar la resta, que sería a través de la sustracción por partes.

B: es una resta, a ver si yo a las 3 y media le quito las 5 me saldrá ¿qué? El tiempo que he estado fuera $\left(\right.$ A.1.2) ${ }^{4}$

[...] B: eran 684 y 186, los que tengan el cuaderno lo hacen, explícaselo J a los que están atendiendo

$J^{5}$ : primero le quitamos a 186 le quitamos 100 (escribe el 100 en el primer cuadrado)

B: ahí pongo 100 y eso se lo quito a los dos (A. 2.1)

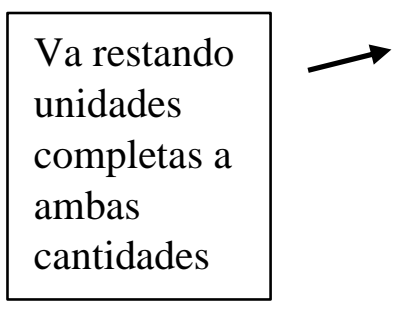

Primera columna: cantidad que va a restar

\begin{tabular}{|l|l|l|}
\hline \multicolumn{2}{|l|}{$684-186$} \\
\hline 100 & 584 & 86 \\
\hline 80 & 504 & 6 \\
\hline 6 & 498 & 0 \\
\hline
\end{tabular}

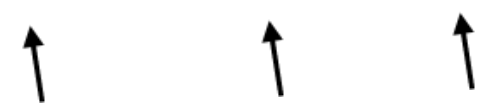

Cuando una de las cantidades llega a cero ya tienen el resultado

Para indagar la posible relación de este conocimiento con su formación en ABN, se le preguntó en la entrevista sobre el significado de la resta para él, Bartolomé contestó lo siguiente: [...] yo mi visión de la resta cambió tras mi formación con el método ABN, restar para mí no es más que comparar una cantidad con otra y ver la diferencia entre ambas.

\footnotetext{
${ }^{2}$ Las unidades de información se han indicado de forma abreviada con una A. de actividad, un primer número que corresponde al número de la actividad explicado anteriormente y un segundo número en función de los indicios encontrados.

${ }^{3}$ Inicial de la intervención de un alumno/a según su nombre.
} 
DOI: $10.20396 /$ zet.v29i00.8661216

Antes de esto el significado que tenía para mí era como algo más mecánico, que si ahora resto y ahora me tengo que llevar no sé cuánto y en este caso comprendes mucho más lo que haces sin llevarte cosas sin saber lo que te llevas.

En relación con el procedimiento de la resta, se parte de una cantidad de forma que el alumno/a tiene que llegar a otra (contar a partir de o escalera ascendente), también se han encontrado evidencias con la realización de la resta por partes; el docente conoce cómo se hace la escalera ascendente.

B: muy bien, dos céntimos. Si yo tengo 4.38 y le añado 2 céntimos ¿a dónde llego I? (I se queda pensativo) Tengo 4.38 y le añado dos céntimos llego a... (A. 3.2)

Tabla 2 - Síntesis de conocimientos del subdomínio KoT

\begin{tabular}{ccc}
\hline & KoT & \\
\hline INDICADORES DE ANÁLISIS & CATEGORÍA & UNIDAD DE INFORMACIÓN \\
\hline Conocer el significado de la resta & Procedimientos & A.1.2 \\
& & A.2.1 \\
Conocer la forma de proceder para & \\
la realización de una resta & A.3.2 \\
\end{tabular}

\section{Análisis del Conocimiento de la Estructura de las Matemáticas (KSM)}

En lo que respecta al subdominio KSM se ha encontrado una evidencia relacionada con una Conexión de Simplificación. El docente utiliza el SND como Conexión de Simplificación que le ayudaría a disminuir la dificultad que supone trabajar con un contenido que no es de este nivel (los decimales).

B: escuchamos que J. acaba de ser sincera y dice que no lo entiende, quizás no es la única persona que no lo entiende. Mirad yo voy a poner en rojo los céntimos y de azul los céntimos, pero de monedas doradas (de la primera rejilla que ponía de 2,82 a 10 dibuja el 8 de rojo y el 2 de azul) (A.4.2)

\begin{tabular}{|c|c|}
\hline \multicolumn{2}{|c|}{ De $2,82 €$ a $10 €$} \\
\hline 0,08 & 2,90 \\
\hline 0,10 & 3,00 \\
\hline 7,00 & 10,00 \\
\hline \multicolumn{2}{|c|}{ Total: $7,18 €$} \\
\hline
\end{tabular}


DOI: $10.20396 /$ zet.v29i00.8661216

Tabla 3 - Síntesis de conocimientos del subdomínio KSM

\begin{tabular}{ccc}
\hline \multicolumn{3}{c}{ KSM } \\
\hline INDICADORES DE ANÁLISIS & CATEGORÍA & UNIDAD DE INFORMACIÓN \\
\hline $\begin{array}{c}\text { Conocer recursos de colores para } \\
\text { el SND para diminuir la dificultad } \\
\text { de los decimales }\end{array}$ & $\begin{array}{c}\text { Conexión de } \\
\text { simplificación }\end{array}$ & A.4.2 \\
& & \\
\hline
\end{tabular}

- Dominio del Conocimiento Didáctico del Contenido (PCK)

Análisis del Conocimiento de las Características de Aprendizaje de las Matemáticas (KFLM)

En este subdominio se han encontrado varias evidencias relacionadas principalmente con las fortalezas y dificultades que presenta un contenido respecto al aprendizaje de los estudiantes, así como una evidencia en la forma de interacción con un contenido matemático.

Cuando el docente realiza la resta por partes, muestra conocer la dificultad que pueden tener los alumnos/as al trabajar con un contenido nuevo (las horas); por ello, decide ir restando con cantidades más pequeñas.

B: atentos, y ahora ojo a lo que voy a hacer los niños en el cuaderno pongo 15:30 menos 17:00 y ahora quito una hora (realiza la rejilla en la pizarra en la que se irá haciendo la resta y solo escribe th en el primer cuadrante de la misma) (A.1.3)

\begin{tabular}{|l|l|l|}
\hline \multicolumn{1}{|c|}{$15: 30-17: 00$} \\
\hline $\boldsymbol{l} \boldsymbol{h}$ & & \\
\hline & & \\
\hline & & \\
\hline
\end{tabular}

En la posterior entrevista, se le preguntó a Bartolomé si se aprovechaba en alguna situación de la libertad que ofrece el uso del cálculo abierto, a esta pregunta nos contestó lo siguiente: "Pues mira a primera hora con un contenido que ellos no conocen o que yo sé que tienen dificultad por lo que sea prefiero restar desde cantidades más pequeñas para que la resta no le suponga una dificultad y se fijen bien en el procedimiento, una vez que ya saben hacerlo en realidad ya pueden restar las cantidades que ellos quieran. Digamos que, como todo y, sobre todo, en los inicios es mejor empezar más poco a poco para adquirir habilidad".

En relación con lo anterior y, con el conocimiento de la resta, Bartolomé cada vez que procede a realizar el cálculo les pregunta a los estudiantes para asegurarse de que entiendan el 
procedimiento, en este caso, sabe que estos tienen dificultades en el procedimiento de la sustracción; estaríamos ante una evidencia sobre el conocimiento de una dificultad que se repite en varias actividades.

[...] si yo le quito una hora, si es una resta ¿qué hago J.? ¿a quién se la quito a las dos horas (a las dos cantidades) o a una? (A.1.4)

B: muy bien le ha quitado 100 a los dos, ahora ¿cuánto le quitamos?

$J: 80$

B: pues venga a las dos cantidades, a los dos, ¿no? Ahí pongo 80 y eso se lo quito a los $\operatorname{dos}($ A.2.2)

Para convertir este indicio en evidencia se le hizo la siguiente pregunta: "el hecho de que emplees cuatro formas para realizar la resta puede llevar a error en el procedimiento por parte de los alumnos, ¿consideras algunas de esas formas más complicada de entender?" su respuesta fue la siguiente: $\mathrm{Si}$ es verdad que, en segundo ciclo, en tercero y cuarto, es cuando dominan perfectamente estas 4 formas de realizar la resta. Pero en un segundo de primaria les cuesta más sobre todo cuando se trata de un problema o de un contenido nuevo como hablábamos antes le cuesta elegir o saber cuál de ellas es la correcta, por mi experiencia desde que las uso para ellos las más fáciles son la escalera ascendente o descendente pero cuando dominan todas no les supone dificultad. Lo que pasa que en segundo de primaria y primero están todavía aprendiéndolas.

Por último, se ha encontrado una evidencia en la que el maestro tiene conocimiento sobre la forma de interacción de los alumnos/as en lo que al cálculo se refiere, en este caso, el docente es consciente de que los niños/as tienen una estrategia personal que les facilita el cálculo ("contar a partir de").

[...] el de la página 98 en vez de contar hasta un euro vamos a tener que llegar a 10 euros. Nos van a dar una cantidad y esa cantidad la vamos a completar con una escalera ascendente vamos a leer la primera parte lo que viene en el cuadradito verde J. ¿Qué dice? Dice llega a 10 euros con escalera ascendente que viene una camiseta. ¿Qué dice el problema?

J: tienes 4,38 euros ¿cuánto te falta para llegar a 10? (A.3.1)

[...] B: muy bien, dos céntimos. Si yo tengo 4.38 y le añado 2 céntimos ¿a dónde llego J? (se queda pensativo) Tengo 4.38 y le añado dos céntimos llego a (A.3.2) 
Tabla 4 - Síntesis de conocimientos del subdomínio KFLM

\begin{tabular}{ccc}
\hline & KFLM & \\
\hline INDICADORES DE ANÁLISIS & CATEGORÍA & UNIDAD DE INFORMACIÓN \\
\hline $\begin{array}{c}\text { Reconocer la facilidade de restar com } \\
\text { cantidades más pequenas em la } \\
\text { iniciación de um contenido }\end{array}$ & $\begin{array}{c}\text { Fortalezas y } \\
\text { dificultades }\end{array}$ & A.1.3 \\
$\begin{array}{c}\text { Conocer las dificultades de los } \\
\text { alunos/as para restar em las dos } \\
\text { cantidades }\end{array}$ & \\
$\begin{array}{c}\text { Conocer uma estratgia de cálculo que } \\
\text { poseen los niños que les facilita su } \\
\text { realización }\end{array}$ & $\begin{array}{c}\text { Formas de interacción } \\
\text { con um contenido } \\
\text { matemático }\end{array}$ & A.1.4 \\
& A.3.1 & A.3.2
\end{tabular}

\section{Análisis del Conocimiento de la Enseñanza de las Matemáticas (KMT)}

En este subdominio es donde más evidencias se han encontrado y, concretamente, dentro de la categoría de Recursos Materiales y Virtuales, la interpretación de este hecho se realizará en la discusión.

En relación con la categoría de Teorías sobre enseñanza situamos el conocimiento de un método alternativo de cálculo (la escalera ascendente) al cálculo convencional y su uso para facilitar la enseñanza.

[...] el de la página 98 en vez de contar hasta un euro vamos a tener que llegar a 10 euros. Nos van a dar una cantidad y esa cantidad la vamos a completar con una escalera ascendente vamos a leer la primera parte lo que viene en el cuadradito verde J. ¿Qué dice? Dice llega a 10 euros con escalera ascendente que viene una camiseta. ¿Qué dice el problema?

J: tienes 4,38 euros ¿cuánto te falta para llegar a 10? (A.3.1)

[...] B: muy bien, dos céntimos. Si yo tengo 4.38 y le añado 2 céntimos ja dónde llego J? (se queda pensativo) Tengo 4.38 y le añado dos céntimos llego a (A.3.2)

En cuanto a los recursos materiales y virtuales, el docente se apoya en diferentes recursos (principalmente materiales) en las explicaciones para facilitar el aprendizaje de la resta. Por tanto, el docente no solo parece conocer estos recursos, sino que también es consciente de su efectividad para la enseñanza. Concretamente los recursos empleados son los siguientes:

- Círculos, semicírculos y cuartos de círculos de cartulina (para las horas, medias horas y cuartos de hora respectivamente).

B: bien pues ahora es el momento de coger con nuestras horas y ponemos al ladito de la pizarra todos los números 3 de las mesas de las bandejas cogéis 3 horas enteras ¿hemos cogido todo el mundo 3 horas? (dibuja al lado de la cuadrícula 3 círculos y un semicírculo) 
DOI: $10.20396 /$ zet.v29i00.8661216

cogéis también media hora (deja tiempo para que lo saquen de la bandeja) ¿todos los grupos han cogido 3 horas y media? (pide que guarden silencio). Ahora R. ¿qué falta por poner del problema ahora? (A.1.1)

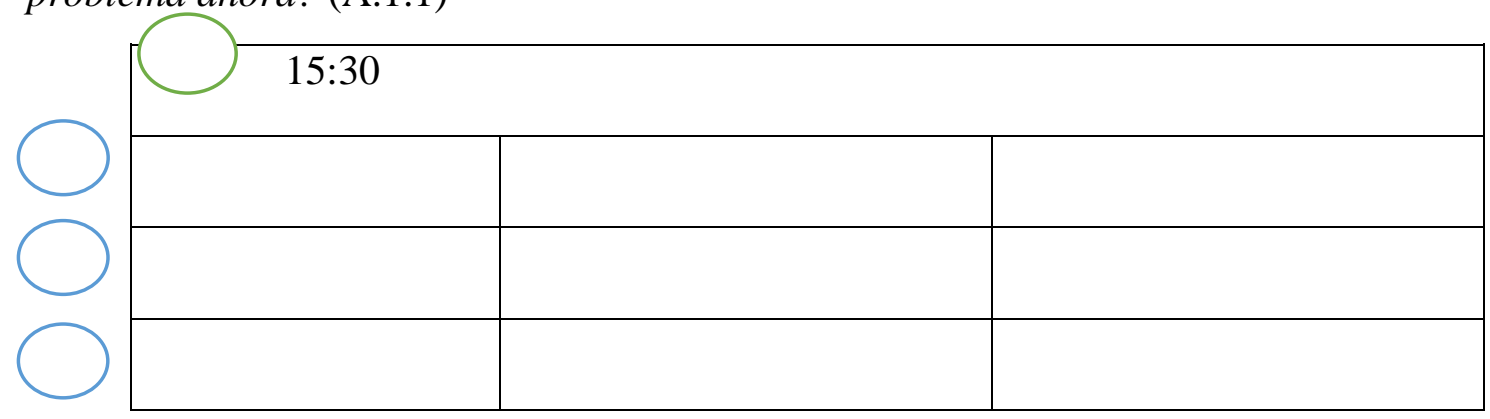

- Monedas de cartón de euro y céntimo para las actividades de transacción.

J: ¿por qué está puesto con comas?

B: porque eso son céntimos J esos son o los de cobre o los dorados (coge una moneda de un euro de cartón y la enseña) esto es un euro, el euro va antes de la coma y de estos tengo 3 (coge en monedas 3 euros y las cuenta) uno, dos y tres. Y ahora tengo 25 (coge una moneda de 20 y una de 5, las suelta) vale yo tengo esto J (le enseña la moneda de 5) me hace falta otros 5 céntimos, si tengo 5 y 5 ¿cuánto tengo? (A.4.1)

B: 70 y 30 céntimos que tenía lo cambio por un euro (suelta la moneda de 50, las dos de 20 y la de 10 y coge una moneda de un euro juntándolas con las otras tres monedas de euro) (A.4.3)

- Colores para el Sistema Numérico Decimal

B: escuchamos que J. acaba de ser sincera y dice que no lo entiende, quizás no es la única persona que no lo entiende. Mirad yo voy a poner en rojo los céntimos y de azul los céntimos, pero de monedas doradas (de la primera rejilla que ponía de 2,82 a 10 dibuja el 8 de rojo y el 2 de azul) (A.4.2)

\begin{tabular}{|c|c|}
\hline \multicolumn{2}{|c|}{ De $2,82 €$ a $10 €$} \\
\hline 0,08 & 2,90 \\
\hline 0,10 & 3,00 \\
\hline 7,00 & 10,00 \\
\hline \multicolumn{2}{|c|}{ Total: $7,18 €$} \\
\hline
\end{tabular}

Por último, encontramos, dentro de la categoría de Estrategias, técnicas, tareas y ejemplos el conocimiento del maestro de una técnica en la que le permite hacer comprender a los estudiantes que la resta es un proceso iterativo y en su realización pueden observar lo que llevan quitado y lo que les queda. 
DOI: $10.20396 /$ zet.v29i00.8661216

B: eran 684 y 186, los que lo tengan el cuaderno la hacen, explícaselo J. a los que están atendiendo

J: primero le quitamos a 186 le quitamos 100 (Escribe el 100 en el primer cuadrado)

B: ahí pongo 100 y eso se lo quito a los dos

(J hace la primera fila) (A.2.1)

\begin{tabular}{|l|c|c|}
\hline \multicolumn{2}{|c|}{$684-186$} \\
\hline 100 & 584 & 86 \\
\hline & & \\
\hline & & \\
\hline
\end{tabular}

Tabla 5 - Síntesis de conocimientos del subdomínio KMT

\begin{tabular}{ccc}
\hline \multicolumn{3}{c}{ KMT } \\
\hline INDICADORES DE ANÁLISIS & CATEGORÍA & UNIDAD DE INFORMACIÓN \\
\hline Conocer y emplear um método & Teoría sobre & A.3.1 \\
alternativo para el cálculo & enseñanza & A.3.2 \\
Conocer la efectividad de & Recursos materiales & A.1.1 \\
elementos materiales para la & y virtuales & A.4.1 \\
mejora de la comprensión de um & & A.4.2 \\
contenido & & A.4.3 \\
Conocer la sustracción de ABN & Estrategias, técnicas, & A.2.1 \\
como técnica para comprender & tareas y ejemplos & \\
que la resta es un processo & & \\
iterativo en el que aparece lo que & & \\
llevo quitado y lo que me queda & &
\end{tabular}

\section{Valoración del análisis}

En esta investigación se han obtenido evidencias relacionadas con el conocimiento especializado del maestro en la resta en relación con los subdominios del MTSK. Cabe destacar que este docente emplea el método $\mathrm{ABN}$ por lo que, a continuación, se discutirá si esos conocimientos que se han analizado anteriormente proceden de su formación en este método, para poder afirmar que estas evidencias encontradas proceden de tal formación nos hemos apoyado en la entrevista realizada al maestro. No se ha podido constatar nuestra valoración de los resultados con otras anteriores ya que no se ha encontrado ningún trabajo que relacione los elementos ABN y MTSK.

En lo que al conocimiento de la resta respecta (subdominio KoT) el docente no solo posee un conocimiento en lo que a la resta se refiere, sino que se basa en el método $A B N$ para su realización. En (A.1.2) muestra que, en el procedimiento llevado a cabo, es indiferente la 
posición de minuendo y sustraendo según sea mayor o menor, así, realiza una resta en la que la cantidad menor viene precedida de la mayor. Cuando se le preguntó desde cuándo utiliza esta forma de realizar la resta en la que no importa la posición del minuendo y sustraendo su respuesta fue:

Si es verdad que con el método tradicional nunca me he planteado restar una cantidad menor delante de la mayor ni mucho menos iba a pensar en que se puede restar de cuatro formas diferentes, en este caso, aunque con la formación en ABN te avisan de que es mejor o más conveniente restar con la cantidad mayor delante, una vez que los alumnos comprenden lo que están haciendo, que están comparando cantidades saben hacerlo de cualquier manera,

lo que muestra la influencia de su formación, en general y cabe conjeturar que en ABN, en prticular, por su dilatada experiencia con el método. Naturalmente, podría argumentarse que su conocimiento en ABN forma parte de su conocimiento especializado.

Además, en este ejemplo, como en otros (A. 2.1), el docente hace la resta por partes, algo característico de $\mathrm{ABN}$, pues se trata de un Algoritmo que permite al alumnado restar cantidades en función de la dificultad personal encontrada. En concreto, en la entrevista se le preguntó acerca de esta manera de realizar la resta, y contestó lo siguiente:

Yo con el método tradicional que era el anterior que usaba a este realizaba el cálculo mecánico tal y como se conoce de este método y nunca me había planteado esta posibilidad, si es verdad que a la hora por ejemplo de corregir actividades pues puede ofrecerte un problema porque rara vez vas a ver que los alumnos resten todas las mismas cantidades, porque se trata de un cálculo abierto y cada uno da los pasos que quiere y resta las cantidades que para él son más fáciles.

Una vez más nos muestra la procedencia de este conocimiento.

En relación con la importancia dada al SND por $\mathrm{ABN}$, parece mostrarse tanto en el Procedimiento llevado a cabo por el maestro en (A.3.2), pues busca redondear a unidades completas, como en el Conocimiento de la Estructura de las Matemáticas (KSM), ya que el docente emplea este SND como Conexión de Simplificación para que los alumnos/as puedan entender un contenido que no es de su nivel (los decimales), y así poder trabajar con el dinero, dándole la importancia de la contextualización de las cifras, algo propio de $\mathrm{ABN}$ (A.4.2). En la entrevista se le realizó una pregunta en la que se le contextualizó esta actividad en concreto en la que se empleó los decimales y se le preguntó sobre a qué se debía la importancia del SND y la respuesta del docente fue:

Pues mira yo nunca me plantee usar decimales en un segundo de primaria, pero si te paras a pensar es que las monedas ellos las conocen, están en su vida diaria, sabe que existen los euros y los céntimos, saben cuántos céntimos tiene un euro y en realidad el sistema numérico decimal lo llevan trabajando del mismo modo casi desde infantil, yo por mi formación, por los vídeos que he visto con ABN a primera hora pensaba ¿pero ¿cómo van a ser capaces de comprender los decimales? Pues si lo comprenden porque son euros y céntimos, cosas de su día a día, si tú te sirves de ese SND te va a ayudar para que ellos lo comprendan porque es algo que también conocen y que con este método se tiene muy trabajado desde que son pequeños. 
En lo que al subdominio del KFLM respecta, y en relación con ABN, el docente realiza la sustracción quitando cantidades más pequeñas, lo hace poco a poco para disminuir la dificultad que pueden tener los alumnos/as ante la primera toma de contacto de un contenido nuevo (A.1.3). Además, dado que en $\mathrm{ABN}$ hay cuatro formas de proceder para la realización de una resta y en una de ellas (la detracción), en su procedimiento, hay que quitar lo mismo a las dos cantidades dadas, el maestro conoce la dificultad que tienen los alumnos/as para llevar a cabo este tipo de sustracción y enfatiza su procedimiento, estaríamos ante una evidencia del conocimiento de la dificultad que poseen los alumnos/as (A.2.2) y (A.1.4). La última evidencia relacionada con este subdominio ha sido la del conocimiento que el docente tiene en la forma de interacción de los alumnos con un contenido matemático, con la escalera ascendente, y que estaría relacionada con "contar a partir de", se trata de una de las cuatro formas de realizar la resta en $\mathrm{ABN}$ (A.3.1) y (A.3.2). En relación con esta técnica en concreto y debido a que, en este caso, ya fue recogida con anterioridad a la aparición de este método, se le preguntó a Bartolomé desde cuándo conocía la técnica de la escalera ascendente. Su respuesta fue la siguiente: hasta que yo no me formé en ABN no conocí estas técnicas, sabía que este método existía, que empleaba otras técnicas, antes a esto yo tenía la formación básica digamos que cualquier docente puede tener en matemáticas, evidenciando de nuevo la procedencia del conocimiento señalado.

En relación con el último subdominio presente en el conocimiento de Bartolomé (KMT), este hace explícita una de las alternativas de cálculo que posee ABN para realizar la resta (la escalera ascendente), mostrando un conocimiento de una estrategia de enseñanza ${ }^{6}$ (A.3.1) y (A.3.2). Además, tanto en esta forma de realizar la resta (escalera ascendente), como en las tres formas restantes indicadas por $\mathrm{ABN}$, le permite al docente, con el uso de la rejilla explicitar el proceso de la resta. Podemos decir, por tanto, que posee una técnica en la que le permite hacer comprender a los alumnos/as todo el proceso de la resta, estos pueden ver lo que llevan quitado y lo que les queda (A.2.1). Con el fin de saber desde cuándo conoce y emplea estos recursos se preguntó si, antes de conocer este método, realizaba restas por partes o conocía o empleaba alguna técnica o recurso para llevar a cabo, en este caso, la sustracción en classe. Su respuesta fue: Yo con el método tradicional he sido bastante rudimentario, me limitaba a las actividades recogidas en el libro y poco más, cuando ya te ofrecen este abanico de posibilidades para enseñar las matemáticas es cuando empecé a usar recursos y a formarme profundamente.

Es destacable, además, la gran cantidad de recursos que el docente emplea para llevar a cabo estas actividades, concretamente, Bartolomé las realiza en contextos con temáticas diferentes (las horas y las monedas) dando importancia a la manipulación y contextualización, tal y como ABN indica. Es de suma importancia para el docente el uso de

\footnotetext{
${ }^{6}$ Estrategia de enseñanza derivada de su conocimiento de la estrategia "contar a partir de" como propia de los niños de esa etapa (Maza, 1991), lo que, de nuevo nos muestra conocimiento de las características del aprendizaje de las matemáticas (KFLM).
} 
Recursos materiales: círculos de cartulina para las horas (A.1.1), monedas de cartón de euros y céntimos para las monedas (A.4.1) y (A.4.2) y colores para facilitar comprensión de contenidos (A.4.3), de los que parece conocer sus potencialidades y limitaciones. Por ello, en la entrevista, a la pregunta sobre la importancia del uso de recursos materiales y de la contextualización el maestro contestó:

mira yo he aprendido y me ha servido mucho los recursos materiales porque si ellos se equivocan no tienen que pensar mentalmente en que se han equivocado, lo tienen delante y reconocen el error, además la manipulación en ABN ya la trabajan desde infantil y esto me ayuda a mí a seguir trabajándola, si es cierto que en cursos más superiores no se emplea tanto, pero que en el inicio del aprendizaje de las matemáticas se pueda aprender manipulando y comprendiendo pues ayuda bastante.

Como puede verse, su formación en $\mathrm{ABN}$ se muestra determinante en su conocimiento.

\section{Conclusiones}

A partir de esta investigación no solo se han identificado conocimientos de los diferentes subdominios del MTSK que pone en juego un maestro cuando enseña la resta, sino que se ha intentado ir más allá, explicitando cuáles de esos conocimientos forman parte de su formación en $\mathrm{ABN}$ en este contenido específico, para ello, se ha tomado como base la información recogida en el marco teórico.

En los resultados se han obtenido evidencias que nos hacen pensar que Bartolomé tiene un ominio en la enseñanza de la resta con este método; concretamente se han obtenido evidencias de los subdominios Conocimiento de los Temas (KoT), Conocimiento de la Estructura Matemática (KSM), Conocimiento de las Características de Aprendizaje de las Matemáticas (KFLM) y, por último, Conocimiento de la Enseñanza de las Matemáticas (KMT). De este último es destacable la gran cantidad de recursos que conoce y emplea para facilitar la comprensión de la resta, este conocimiento conjeturamos que le viene dado por su formación con un método donde se enfatiza la importancia de uso de materiales para contextualizar los contenidos.

También podemos afirmar que su amplio conocimiento de ABN puede justificar la interrelación existente entre los conocimientos de los diferentes subdominios que hemos identificado. En relación con el contenido de la resta, que le han dotado de conocimientos y dominios de los diferentes procedimientos para llevarla a cabo (KoT). También le han permitido conocer, tanto las características de enseñanza para la misma, con el uso de recursos, técnicas o teorías que potencien y faciliten la comprensión (KMT), como las del aprendizaje de los alumnos/as en cuanto a las dificultades que podrían presentar o a la forma de interactuar con el contenido (KFLM), un dominio suficiente como para, además, poseer conexiones entre contenidos que le facilitan la enseñanza de la resta (KSM). 
En este sentido, consideramos que se ha dado respuesta a las preguntas de investigación planteadas, pues, se ha pretendido conocer el conocimiento especializado de un maestro de Primaria cuando trabaja la resta en segundo curso y comprender cuales de esos conocimientos que se han expuesto provienen de su formación en $\mathrm{ABN}$, considerando que el docente posee un amplio dominio en la resta y que su forma de trabajarla se debe a la formación con el método.

Cabe destacar que han sido cuatro los subdominios que se han encontrado, que evidencian dicho conocimiento (los expuestos anteriormente); sin encontrar evidencias de los subdominios KPM y KMLS, quizás se deba al instrumento de recogida de información utilizado (la observación). También sería interesante un estudio comparativo de caso, en contextos similares, con otro maestro segundo de Primaria no familiarizado con ABN, para para poder seguir explorando acerca del potencial de la formación en $A B N$ en la configuración del conocimiento especializado mostrado.

Es obvio que estos conocimientos pueden proceder de otras fuentes, incluso de su propia experiencia, pero no es menos cierto que nuestro informante reconoce explícitamente su procedencia en $\mathrm{ABN}$.

\section{Agradecimientos}

El Gobierno de España (EDU2013-44047-P y EDU2016-81994-REDT), y el centro de investigación COIDESO (Universidad de Huelva. España) apoyan esta investigación.

\section{Referencias}

Ablewhite, R. C., y Paret, A. M. L. (1971). Las matemáticas y los menos dotados. Madrid: Morata.

Aguilar, A., Carreño, E., Carrillo, J., Climent, N., Contreras, L., Escudero, D., ... Rojas, N. (2013). El Conocimiento Especializado del Profesor de Matemáticas: MTSK. Actas del VII CIBEM (pp.5063- 5069). Montevideo: CIBEM.

Ball, D. L., Thames, M.H., \& Phelps, G. (2008). Content Knowledge for teaching: What makes it special? Journal of teacher education, 59, 389- 407.

Bassey, M. (1999). Case study research in educational settings. Buckingham: McGraw-Hill Education.

Bracho-López, R. (2013). Menos reglas y más sentido: alternativas metodológicas a los algoritmos de cálculo tradicionales para el desarrollo del sentido numérico en la educación primaria. Actas del VII CIBEM (pp. 70-77). Montevideo: CIBEM.

Bracho-López, R., Adamuz-Povedano, N., Gallego-Espejo, M. C., \& Jiménez-Fanjul, N. (2014). Alternativa metodológica para el desarrollo integral del sentido numérico en niños y niñas de primer ciclo de educación primaria. In M. T. González, M. Codes, D. Arnau and T. Ortega (Eds.), Investigación en Educación Matemática XVIII (pp. 161176). Salamanca: SEIEM. 
Bracho-López, R., Adamuz-Povedano, N., Jiménez-Fanjul, N., \& Gallego-Espejo, M. C. (2014). Una experiencia de investigación-acción colaborativa para el desarrollo del sentido numérico en los primeros años de aprendizaje matemático. In J. L. González, J. A. Fernández-Plaza, E. Castro-Rodríguez, M. T. Sánchez-Compaña, C. Fernández, J. L. Lupiáñez and L. Puig (Eds.), Investigaciones en Pensamiento Numérico y Algebraico e Historia de las Matemáticas y Educación Matemática (pp. 1-9). Málaga: Departamento de Didáctica de las Matemáticas, de las Ciencias Sociales y de las Ciencias Experimentales y SEIEM.

Bracho-López, R., Gallego-Espejo, M., Adamuz-Povedano, N., \&Jiménez-Fanjul, N. (2014). Impacto escolar de la metodología basada en algoritmos ABN en niños y niñas de primer ciclo de Educación Primaria. Revista Iberoamericana de Educación Matemática, 39, 97 109.

Brown, R., y Burton, R. (1978). Diagnostic models for procedural in basic mathematical skills. Cognitive Science, 2, 155-192.

Canto, M.C. (2014). Curso: Método ABN. Primer ciclo (Por unas matemáticas sencillas, naturales y divertidas). Ronda: CEP de Ronda. Recuperado de https://calculoabn.com/0.1/wordpress/4.7.z/wp-content/uploads/abn-primer-ciclo-2.pdf

Carpenter, T., Franke, M., Jacobs, V., \& Fennema, E. (1996). Invention and understanding in the development of multidigit addition and subtraction procedures: A longitudinal study. Annual meeting of the American Research Association: New York.

Carrillo, J., Climent, N., Contreras, L. C., \& Muñoz-Catalán, M. D. C. (2013). Determining specialised knowledge for mathematics teaching. In B. Ubuz, et al (Ed.), VIII Congress of the European Society for Research in Matehematics Education (CERME 8) (pp. 29852994). Antalya, Turkey: Middle East Technical University, Ankara.

Carrillo, J., Climent, N., Montes, M., Contreras, L.C., Flores-Medrano, E., Escudero-Ávila, D., Vasco-Mora, D., Rojas, N., Flores, P., Aguilar-González, A., Ribeiro, M., \& MuñozCatalan, M.C. (2018). The Mathematics Teacher's Specialised Knowledge (MTSK) model. Research in Mathematics Education, 20(3), 236-253. https://doi.org/10.1080/14794802.2018.1479981

Carrillo, J., Contreras, L.C., \& Montes, M. (2013). Conocimiento del profesor de Matemáticas: Enfoques del MKT y del MTSK. In A. Berciano, G. Gutiérrez, A. Estepa and N. Climent (Eds.), Investigación en Educación Matemática XXI (pp. 404-410). Bilbao: SEIEM.

Castro, E., Rico, L., \& Castro, E. (1987). Números y operaciones. Fundamentos para una aritmética escolar. Madrid: Síntesis.

Fuson, K. (1992). Research on Whole Number Addition and Subtraction. In D. A. Grouws (Ed.), Handbook of Research on Mathematics Teaching and Learning: a project of the National Council of Teachers of Mathematics (pp. 243-275). New York: Maxwell Macmillan International.

Martínez, J. (2011). El método de cálculo abierto basado en números (ABN) como alternativa de futuro respecto a los métodos tradicionales cerrados basados en cifras (CBC). Bordón. Revista de pedagogía, 63 (4), 95-110. 
DOI: $10.20396 /$ zet.v29i00.8661216

Martínez, J. (2018). El cálculo ABN. Un enfoque diferente para el aprendizaje del cálculo y las matemáticas. Padres y Maestros/Journal of parents and teachers, 376, 52-59.

Maza, C. (1991). La enseñanza de la suma y de la resta. Madrid: Síntesis.

Muñoz Catalán, M. C. (2009). El desarrollo profesional en un entorno colaborativo centrado en la enseñanza de las matemáticas: el caso de una maestra novel. Tesis doctoral. Universidad de Huelva, Huelva.

Stake, R. E. (2005). Qualitative Case Studies. En N. K. Denzin y Y. S. Lincoln (Eds.), The Sage handbook of qualitative research (p. 443-466). London: Sage.

Shulman, L. S. (1986). Those Who Understand: Knowledge growth in teaching. Educational Research, 15 (2), 4-14.

Shulman, L. S. (1987). Conocimiento y enseñanza. Harvard Educational Review, 57(1), 163196. 